\title{
Cytokine secretion and pyroptosis of cholesteatoma keratinocytes mediated by AIM2 inflammasomes in response to cytoplasmic DNA
}

\author{
CHEN ZHANG ${ }^{1,2^{*}}$, MIN CHEN $^{1,2^{*}}$ and ZHANGCAI CHI ${ }^{1,2}$ \\ ${ }^{1}$ ENT Institute and Department of Otolaryngology, Eye and ENT Hospital, Fudan University; \\ ${ }^{2}$ NHC Key Laboratory of Hearing Medicine, Fudan University, Shanghai 200031, P.R. China
}

Received August 13, 2020; Accepted February 18, 2021

DOI: $10.3892 / \mathrm{mmr} .2021 .11983$

\begin{abstract}
Cholesteatoma constitutes an acquired benign epidermal non-permanent bone lesion that is locally destructive and patients often relapse. Inflammasomes, which mediate the maturation and production of IL-18 and IL-1 $\beta$, resulting in pyroptosis, have been documented to serve a core function in multiple inflammatory conditions. Absent in melanoma 2 (AIM2) is an inflammasome that identifies cytoplasmic DNA and has previously been reported as a pivotal modulator of inflammatory responses. Therefore, the present study aimed to determine the expression levels of AIM2 in human cholesteatoma tissues, and elucidate its function in modulating cytokine production. The expression levels of IL-18, apoptosis-associated speck-like protein containing a CARD (ASC), IL-1 $\beta$, AIM2 and caspase-1 were markedly elevated in cholesteatoma tissues. Protein expression levels of AIM2, caspase-1 and ASC were localized in the cellular cytoplasm, primarily in the granular and prickle-cell layers in the cholesteatoma epithelium. Induction using IFN- $\gamma$, as well as cytoplasmic DNA markedly activated the AIM2 inflammasome and elevated the release of IL-18 and IL-1 $\beta$ in human cholesteatoma keratinocytes. IFN- $\gamma$ was found to enhance poly(dA:dT)-induced pyroptosis of cells and cytokine production. The results of the present study revealed that AIM2 expressed in human cholesteatoma serves a vital function in the inflammatory response by initiating the inflammasome signaling cascade in cholesteatoma.
\end{abstract}

Correspondence to: Dr Zhangcai Chi, ENT Institute and Department of Otolaryngology, Eye and ENT Hospital, Fudan University, 83 Fenyang Road, Shanghai 200031, P.R. China

E-mail: 10111260012@fudan.edu.cn

*Contributed equally

Key words: cholesteatoma, absent in melanoma 2, inflammasome, cytoplasmic DNA, cytokine, pyroptosis

\section{Introduction}

Cholesteatoma constitutes an acquired benign epidermal non-permanent bone lesion that is locally destructive, and patients often relapse (1). It is characterized by squamous epithelium overgrowth with hyper-keratin and bone erosion in the middle ear (1). Regardless of whether cholesteatomas are congenital or acquired, cholesteatomas that are infected appear to become enlarged more promptly and damage local structures, as detected by clinical observation (2). Previous reports have stated that infection may facilitate the aggressiveness of cholesteatoma, and that the subsequent inflammatory response may be the most critical factor in cholesteatoma-induced bone destruction (2-4).

The mucosal innate immune system actively contributes to the host response to bacterial pathogen invasions and cell stress signals via pathogen recognition involving pathogen-associated molecular pattern recognition receptors (PRRs), and the ensuing pro-inflammatory cytokine expression and production (5-7). PRRs constitute several families, such as Toll-like receptors (TLRs) (8) and Nod-like receptors (NLRs) (9). Notably, TLRs $(2,10)$ and NLRs $(11)$ are central for IL-1 $\beta$ release, which plays a core function in inflammatory middle ear disease pathogenesis; NLR family pyrin domain containing 3 (NLRP3) (12) in particular is associated with human middle ear cholesteatoma pathogenesis by modulating the activity of IL-1 $\beta$ via the generation of inflammasomes.

Inflammasomes are a group of intracellular protein complexes that identify multiple inflammation-triggering stimuli and induce pro-inflammatory cytokine maturation, including the maturation of IL-1 $\beta$, as well as IL-18 (13). In the past decade, NLRP3 has been reported to induce the generation of inflammasomes and be activated in cholesteatoma (12). However, to the best of our knowledge, little is currently known regarding its function and whether other inflammasomes participate in inflammatory reactions and the subsequent release of cytokines in cholesteatoma. Besides NLRs, the hematopoietic IFN-inducible nuclear proteins with a 200 amino acid repeat (HIN-200) family member absent in melanoma 2 (AIM2) has previously been documented to activate an inflammasome that is pivotal for inducing caspase- 1 in multiple immune cells in response to cytoplasmic DNA (14-16). 
AIM2 constitutes an IFN-inducible HIN-200 family member with an amino-terminal pyrin domain (PYD) and a carboxy-terminal HIN-200 oligonucleotide/oligosaccharide-binding domain (15). Cytoplasmic DNA recognition by the HIN-200 domain mediates the generation of the AIM2 inflammasome by activating AIM2 oligomerization and mobilization of apoptosis-associated speck-like protein containing a CARD (ASC), which initiates caspase-1 and modulates the release and bioactivity of the IL-1 family of cytokines $(15,16)$. In our previous study, elevated apoptotic cells and extracellular DNA fragments were observed in the cholesteatoma epithelium (17). Furthermore, AIM2 has been demonstrated to regulate IL-1 $\beta$ production in human keratinocytes in response to cytoplasmic double stranded (ds)DNA $(18,19)$. Therefore, the present study hypothesized that human cholesteatoma epithelium may express AIM2, and that cholesteatoma keratinocytes may release IL-1 $\beta$ by generating inflammasomes in response to cytoplasmic DNA.

To test this hypothesis, the present study examined the prospective expression of AIM2 in human cholesteatoma tissues, and then investigated the mechanism underlying the AIM2 inflammasome-mediated secretion of IL-1 $\beta$ in cholesteatoma keratinocytes. The present study aimed to provide a basis for further evaluation into the prospective function of AIM2 in the apoptosis of human cholesteatoma keratinocytes.

\section{Materials and methods}

Tissue samples. Cholesteatoma tissues were acquired from the medial ear of nine subjects (six male and three female patients; age range, 8-52 years; median age, 32 years) during tympanoplasty between January 2018 and December 2018 in the Department of Otolaryngology Head and Neck Surgery, Eye and ENT Hospital of Fudan University (Shanghai, China). The inclusion criteria were as follows: i) pathology consistent with cholesteatoma; ii) for congenital cholesteatoma, intact tympanic membrane and no history of otorrhea. Patients were divided into congenital (three cases; age, 8-14 years) and acquired (six cases; age, 33-52 years) groups. The subjects with congenital cholesteatoma were selected carefully as per the criteria of Derlacki and Clemis (20). The cholesteatoma diagnosis was confirmed by histopathological examination. Small biopsy-acquired samples from the external canal skin of five individuals undergoing tympanoplasty for chronic otitis media were utilized as the control group. Signed informed consent was obtained from all participants; if a patient was $<18$ years old, informed consent was obtained from a parent or guardian. The study protocols were approved by the Research Ethics Committee of the Eye and ENT Hospital of Fudan University (approval no. 2017024).

Immunofluorescent staining for cholesteatoma tissues. Fixation of the samples in $4 \%$ paraformaldehyde was performed for $24 \mathrm{~h}$ at room temperature, before embedding in paraffin. Serial segments were sliced ( $7-\mu \mathrm{m}$ thick) and fixed on 3-aminopropyltriethoxysilane-coated glass slides. Immunofluorescence was then performed in order to investigate the expression levels of IL-18, ASC, AIM2, caspase-1 and IL-1 $\beta$ in tissue sections. Briefly, blocking of the segments was performed for $1 \mathrm{~h}$ in $10 \%$ normal goat serum (cat. no. ab7481;
Abcam) after deparaffinization and rehydration in graded alcohol series at room temperature. After rinsing, segments were incubated overnight at $4^{\circ} \mathrm{C}$ with the working solution of antibodies against IL-1 $\beta$ (1:200; cat. no. GTX100793), caspase-1 (1:200; cat. no. GTX101322), AIM2 (1:100; cat. no. GTX116487), ASC (1:200; cat. no. GTX102474) and IL-18 (1:250; cat. no. GTX32675) (all from GeneTex, Inc.). The segments were then incubated with Alexa 546-labeled (1:500; cat. no. 715-025-150; Jackson ImmunoResearch) or Alexa 488-labeled (1:500; cat. no. 111-545-144, Jackson ImmunoResearch) goat anti-rabbit antibodies at room temperature for $1 \mathrm{~h}$, and subsequently stained with blue fluorescent DAPI (1:500; cat. no. 1217226; Invitrogen; Thermo Fisher Scientific, Inc.) at room temperature for $15 \mathrm{~min}$. Images were captured using the Axioscope microscope (Carl Zeiss AG).

Cell culture. The isolation and characterization of cholesteatoma keratinocytes was performed as previously described (15). Briefly, cholesteatoma tissue was obtained and delivered to the laboratory following surgical resection. Then, tissues were cut into small segments using scissors, followed by $4^{\circ} \mathrm{C}$ overnight-digestion of the pieces using collagenase IV $(200 \mathrm{U} / \mathrm{ml}$; Sigma-Aldrich; Merck KGaA). The digested segments were rinsed twice using Hanks' Balanced Salt Solution, and then centrifuged for $5 \mathrm{~min}$ at $350 \mathrm{x} \mathrm{g}$ at room temperature. The supernatant was discarded, and $10 \mathrm{ml}$ keratinocyte serum-free medium (KSFM; Invitrogen; Thermo Fisher Scientific, Inc.) enriched with $500 \mathrm{U} / \mathrm{ml}$ penicillin/streptomycin (Invitrogen; Thermo Fisher Scientific, Inc.) was added, followed by culture in a $5 \% \mathrm{CO}_{2}$ humidified atmosphere at $37^{\circ} \mathrm{C}$. The KSFM with the antibiotics was replaced every 3 days. The cell cultures between the third and fourth passages were utilized in the present study.

TUNEL assay. The in situ cell death detection kit (Roche Diagnostics) was utilized in the TUNEL assay to examine DNA fragmentation according to the manufacturer's instructions. Briefly, the Lab-Tech chamber slide, acquired from Nalge Nunc International (Thermo Fisher Scientific, Inc.), was used to culture the cholesteatoma keratinocytes. After that, the cells were incubated with $2 \mu \mathrm{g} / \mathrm{ml}$ poly(dA:dT; cat. no. tlrl-patn; InvivoGen) for $24 \mathrm{~h}$ after priming with IFN- $\gamma$ ( $25 \mathrm{ng} / \mathrm{ml}$; cat. no. 300-02-20; PeproTech) for $24 \mathrm{~h}$, and then the medium was replaced. Another group of cells was treated with only $2 \mu \mathrm{g} / \mathrm{ml}$ poly(dA:dT). After stimulation, cells were fixed for $30 \mathrm{~min}$ using $4 \%$ paraformaldehyde at room temperature, followed by permeabilization in PBS with $0.3 \%$ Tween (PBST) for $5 \mathrm{~min}$ at room temperature. Subsequently, the cells were rinsed using PBS three times, and the chamber slide and the TUNEL reaction were mixed with terminal deoxynucleotidyl transferase (TdT) and TMR-dUTP, and incubated in a humidified environment for $1 \mathrm{~h}$. The slides were then rinsed three times and analyzed using an Axioscope microscope (Carl Zeiss AG). DAPI (1:500) was used to perform the nuclear counterstaining at room temperature for $15 \mathrm{~min}$. The TUNEL ratio was calculated by assessing five randomly selected fields of view $(1: 100)$.

Western blotting. Cholesteatoma keratinocytes were placed in a petri plate $(35-\mathrm{mm})$ at a density of $1 \times 10^{5}$ cells per plate. 
Subsequently, varying titers of IFN- $\gamma$ alone or poly(dA:dT) $(2 \mu \mathrm{g} / \mathrm{ml})$ after priming with IFN $-\gamma(25 \mathrm{ng} / \mathrm{ml})$ for $24 \mathrm{~h}$ were added. After being stimulated for $24 \mathrm{~h}$, the culture supernatants were collected, and the cells were isolated using cell lysis buffer (Beyotime Institute of Biotechnology) and prepared in order to purify the total cellular protein. The protein concentration was determined by BCA protein assay kit (Beyotime Institute of Biotechnology). Equal amounts of total protein $(30 \mu \mathrm{g})$ were separated via SDS-PAGE (12\% gel), and subsequently transferred onto a PVDF membrane (Beyotime Institute of Biotechnology). The membranes were washed with Tris-buffered saline-Tween-20 (20 mM Tris, $500 \mathrm{mM} \mathrm{NaCl}$ and $0.1 \%$ Tween-20) and blocked at room temperature for $1 \mathrm{~h}$ in western blocking buffer (Beyotime Institute of Biotechnology). After that, the membrane-bound proteins were conjugated with the indicated primary antibodies via overnight incubation at $4^{\circ} \mathrm{C}$. Subsequently, conjugation with the peroxidase-labeled secondary antibodies (1:2,500; cat. no. GAR007; Multi Sciences) was performed according to the manufacturer's protocols. Anti- $\beta$-actin was used as the internal loading control. Semi-quantification of the proteins was performed using ImageJ software (version 1.63; National Institutes of Health). The fold-changes were calculated as the ratios of band densities of target protein to $\beta$-actin. The primary antibodies were as follows: Anti-cleaved-caspase-1 (1:1,000; cat. no. GTX134551), anti-AIM2 (1:1,000; cat. no. GTX116487), anti-ASC (1:1,000; cat. no. GTX102474), anti-caspase-1 (1:1,000; cat.no.GTX101322), anti-gasdermin D (GSDMD; 1:500; cat. no. GTX116840), anti-IL-18 (1:1,000; cat. no. GTX32675), anti-IL-1 $\beta$ (1:1,000; cat. no. GTX100793; all GeneTex, Inc.), anti-cleaved N-terminal GSDMD (GSDMD-N; $1: 1,000$; cat. no. ab215203; Abcam) and anti- $\beta$-actin $(1: 2,500$; cat. no. AF0003; Beyotime Institute of Biotechnology).

Statistical analysis. The data represent $\geq 3$ independent assays, with each experiment performed in triplicate, and are presented as the mean \pm standard deviation. One-way ANOVA followed by Bonferroni's multiple comparison test were used for the statistical analysis. $\mathrm{P}<0.05$ was considered to indicate a statistically significant difference.

\section{Results}

Human cholesteatoma tissues express AIM2. In order to investigate whether AIM2 is expressed by human cholesteatoma tissues, the present study assessed the expression of the AIM2 protein in cholesteatoma tissues using both immunofluorescence and western blotting. AIM2 protein was expressed in the cytoplasm of cells, primarily in granular cells, as well as the prickle-cell sheets in the cholesteatoma epithelium (Fig. 1A). Moreover, the AIM2 expression intensity was generally decreased in the basal and parabasal layer (Fig. 1A). However, the western blotting data revealed that the expression of AIM2 varied in these specimens (Fig. 1B and C). The AIM2 content was significantly elevated in acquired cholesteatoma compared with the congenital cholesteatoma (Fig. 1D).

Protein expression of inflammasome constituents is increased in cholesteatoma tissues. As common components of the inflammasome, the expression levels of ASC and caspase-1 proteins were similarly higher in human cholesteatoma tissues. ASC and caspase-1 were localized in the cytoplasm of cells, primarily in granular and prickle-cell sheets in the cholesteatoma epithelium (Fig. 1E and F). The western blotting results revealed that the ASC and caspase-1 protein expression levels in acquired cholesteatoma (Fig. $1 \mathrm{C}$ and $\mathrm{H}$ ) were markedly higher than those in congenital cholesteatoma (Fig. 1B and G). As shown in Fig. 1I, these differences were statistically significant. The present study also observed markedly higher IL-1 $\beta$ and IL-18 protein expression levels in acquired cholesteatoma tissues (Fig. 1G and H). However, the concentrations of IL-1 $\beta$ and IL-18 did not differ significantly between the acquired and congenital cholesteatoma (Fig. 1I). These data suggested that there was enhanced inflammasome activation in the cholesteatoma epithelium.

Expression of AIM2 in cholesteatoma keratinocytes is strongly induced by IFN- $\gamma$. In order to further investigate the effect of IFN- $\gamma$ on the expression of AIM2 in cholesteatoma keratinocytes, varied titers of IFN- $\gamma$ were introduced to cholesteatoma keratinocytes for $24 \mathrm{~h}$, and then the AIM2 protein expression levels were assessed via western blotting. Untreated cholesteatoma keratinocytes expressed low levels of AIM2 protein. At the same time, it was observed that AIM2 protein expression was increased following IFN- $\gamma$ administration (Fig. 2A and B). Based on these data, the treatment of cholesteatoma keratinocytes with $25 \mathrm{ng} / \mathrm{ml}$ IFN- $\gamma$ for $24 \mathrm{~h}$ was established as the optimum concentration for the downstream assays. Collectively, these findings implied that IFN- $\gamma$ induced and upregulated AIM2 expression in cholesteatoma keratinocytes.

Effect of induction of $I L-1 \beta$ and $I L-18$ release on activation of the AIM2 inflammasome in cholesteatoma keratinocytes in response to cytoplasmic DNA. A previous study documented a marked increase in AIM2 expression following induction with IFN- $\gamma$ (21). Similarly, induction with IFN- $\gamma$ $(25 \mathrm{ng} / \mathrm{ml})$ or poly $(\mathrm{dA}: \mathrm{dT})$ after priming with IFN- $\gamma$ $(25 \mathrm{ng} / \mathrm{ml})$ for $24 \mathrm{~h}$ upregulated the protein expression levels of pro-caspase-1 and ASC, which are both constituents of AIM2 inflammasomes (Fig. 3). However, the present study also established that IFN- $\gamma$ actually primed inflammasomes rather than activated them, since the protein expression of the inflammasome constituents was markedly raised in human cholesteatoma keratinocytes, but without any alterations in cleaved-caspase-1, IL-1 $\beta$ or IL-18 titers (Fig. 3A and B). Hence, an AIM2 ligand, poly(dA:dT), was utilized to elevate AIM2 inflammasome activation in cholesteatoma keratinocytes. Treatment with poly(dA:dT) activated the protein contents of cleaved-caspase-1, and the production of IL- $1 \beta$ and IL-18 in IFN- $\gamma$-primed cholesteatoma keratinocytes, relative to the untreated IFN- $\gamma$-primed cells (Fig. 3A and B). These data demonstrated that poly(dA:dT) induced IL-1 $\beta$ and IL-18 production via the activation of caspase-1-dependent AIM2 inflammasomes in cholesteatoma keratinocytes.

Pyroptosis of cholesteatoma keratinocytes on the activation of the AIM2 inflammasome. Pyroptosis is regarded as a pivotal underlying mechanism of active cytokine production caused by alterations in membrane permeability $(22,23)$. As indicated 
A
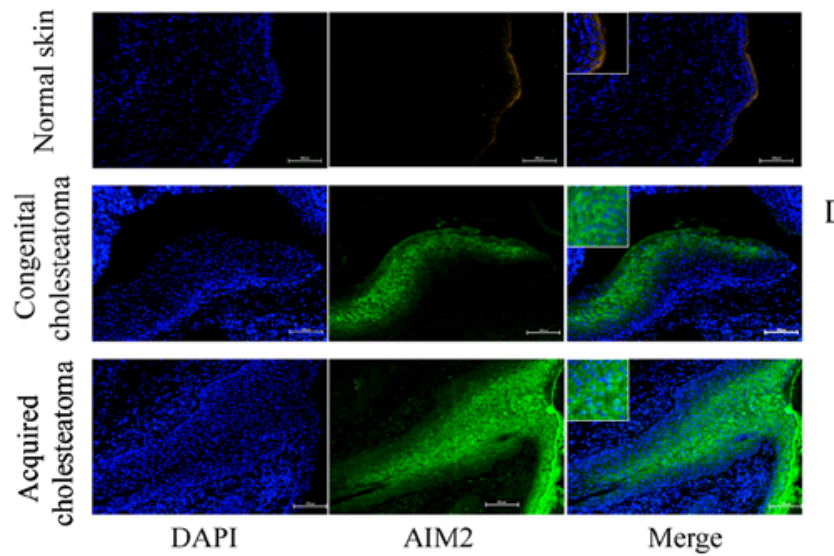

E

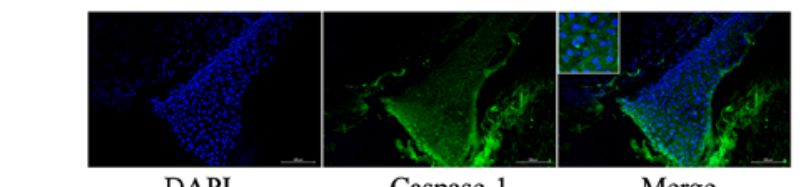

DAPI

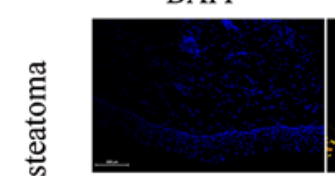

DAPI

Caspase-1

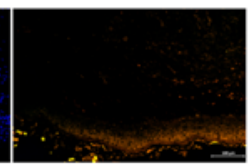

ASC

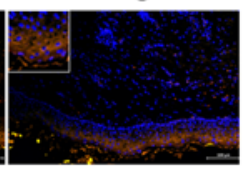

Merge
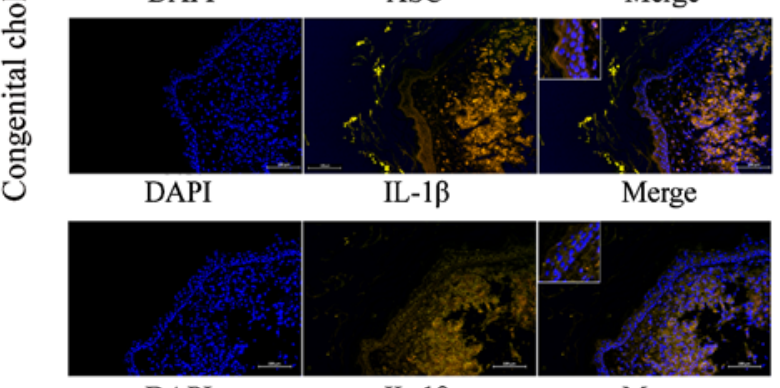

DAPI

G

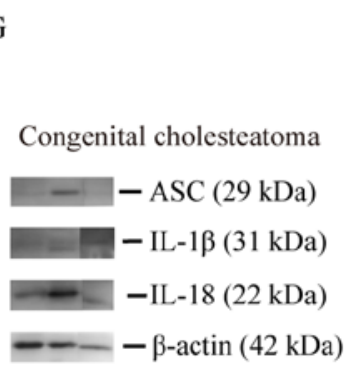

B

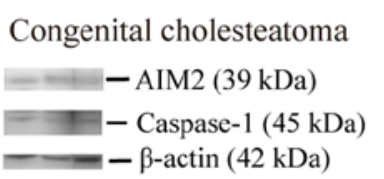

C

Acquired cholesteatoma

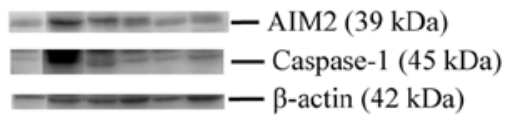

$\mathrm{D}$
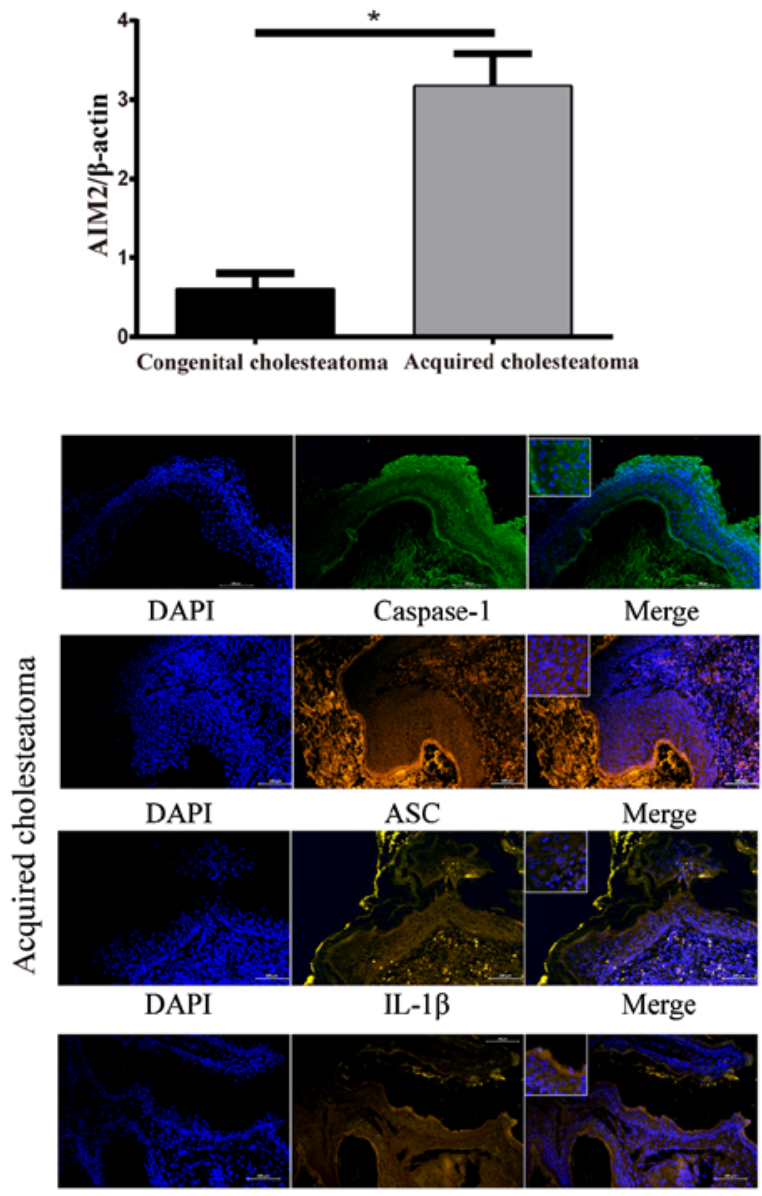

DAPI

IL-18

Merge

I

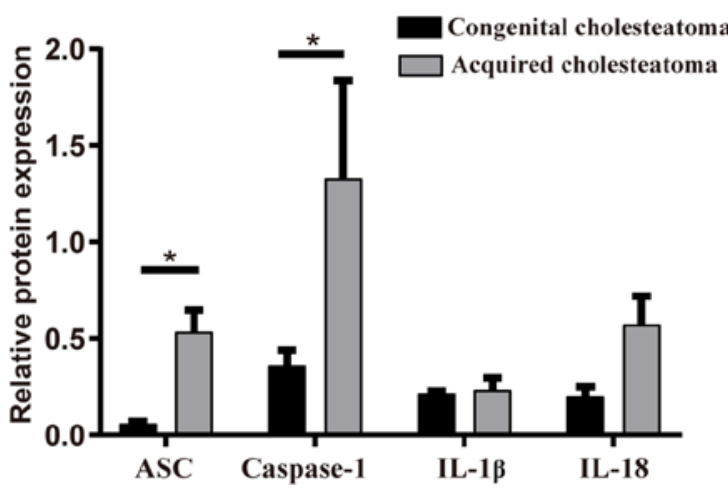

Figure 1. Immunofluorescence and western blotting analyses of inflammasome components in human congenital and acquired cholesteatoma tissues (A) Localization and expression of AIM2 in cholesteatoma tissues and healthy skin tissues. Immunofluorescence analysis revealed that AIM2 was localized in the cytoplasm of cells, primarily in granular and prickle-cell sheets. The nucleus was labelled using DAPI (magnification, x200). (B and C) Western blotting images of AIM2 and caspase-1. (D) Corresponding semi-quantitative analysis of the AIM2 protein content in congenital and acquired cholesteatoma tissues. Localization of ASC, caspase-1, IL-1 $\beta$ and IL-18 in (E) congenital cholesteatoma tissues and (F) acquired cholesteatoma tissues. The immunofluorescence assay revealed that ASC and caspase-1 were mainly localized in the cytoplasm of cells, primarily in granular and prickle-cell layers. The nucleus was labelled using DAPI (magnification, x200). ( $\mathrm{G}$ and H) Western blotting images and (I) corresponding semi-quantitative analysis of the ASC, caspase-1, IL-1 $\beta$ and IL-18 protein expression levels in congenital and acquired cholesteatoma tissues. The relative protein expression content was standardized to $\beta$-actin. The columns indicate the mean \pm standard deviation values ( $\mathrm{n}=3$ per group). ${ }^{*} \mathrm{P}<0.05$. ASC, apoptosis-associated speck-like protein containing a CARD; AIM 2 , absent in melanoma 2.

in Fig. 4A and B, the results of the TUNEL assay demonstrated an increased percentage of apoptotic cells in the poly(dA:dT) and IFN- $\gamma+$ poly $(\mathrm{dA}: \mathrm{dT})$ groups relative to the control. The results in Fig. 4C and D also demonstrated that there was an 

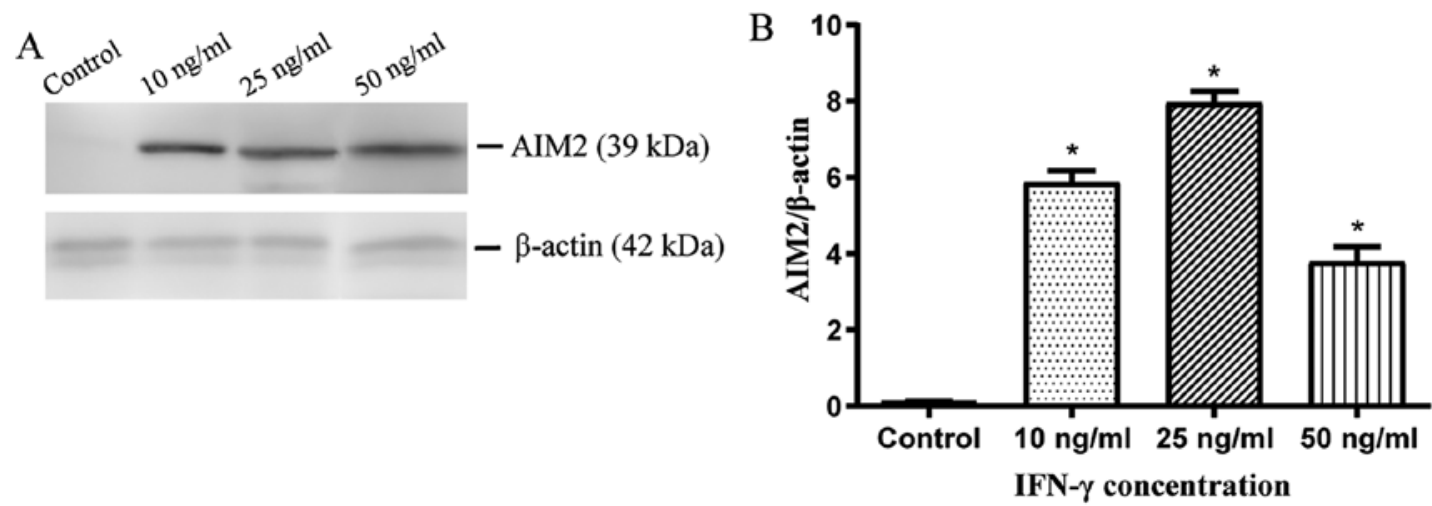

Figure 2. IFN- $\gamma$ induces the expression of AIM2 in human cholesteatoma keratinocytes. (A) Cholesteatoma keratinocytes were treated with different titers of IFN- $\gamma$ for $24 \mathrm{~h}$. The expression levels of AIM2 were assessed using western blotting. (B) The panels indicate the ratios of the band densities of AIM2 to $\beta$-actin. The results are indicated as the mean \pm standard deviation of three different experiments ( $\mathrm{n}=3$ per group). ${ }^{*} \mathrm{P}<0.05$ vs. control group. AIM 2 , absent in melanoma 2.

A

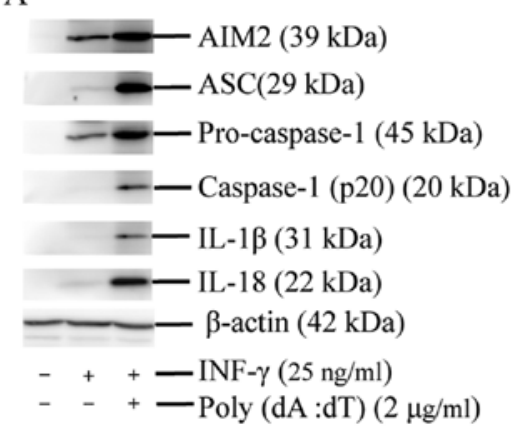

B

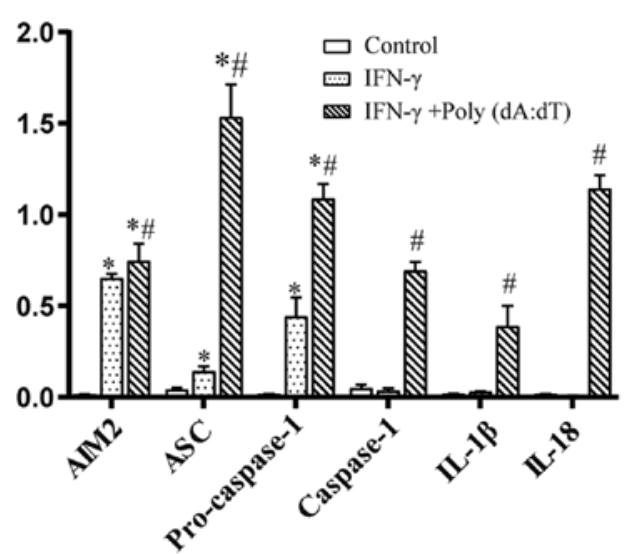

Figure 3. Expression of inflammasome components in human cholesteatoma keratinocytes following stimulation by cytoplasmic DNA after priming with or without IFN- $\gamma(25 \mathrm{ng} / \mathrm{ml})$. (A) Changes in AIM2, ASC, caspase-1, cleaved-caspase-1, IL-1 $\beta$ and IL-18 protein expression levels were determined by western blotting after stimulation with $25 \mathrm{ng} / \mathrm{ml} \mathrm{IFN}-\gamma$ or $25 \mathrm{ng} / \mathrm{ml} \mathrm{IFN}-\gamma+2 \mu \mathrm{g} / \mathrm{ml}$ poly(dA:dT) for $24 \mathrm{~h}$. (B) Semi-quantification of western blotting results. The columns represent the mean \pm standard deviation values $\left(\mathrm{n}=3\right.$ per group). Data were collected from three independent assays. ${ }^{*} \mathrm{P}<0.05$ vs. control group; ${ }^{\#} \mathrm{P}<0.05$ vs. IFN- $\gamma$ group. AIM2, absent in melanoma 2; ASC, apoptosis-associated speck-like protein containing a CARD.

increase in the expression levels of GSDMD-N (31 kDa) in the poly $(\mathrm{dA}: \mathrm{dT})$ and IFN $-\gamma+$ poly $(\mathrm{dA}: \mathrm{dT})$ groups when compared with the blank controls.

\section{Discussion}

To the best of our knowledge, the present study was the first to demonstrate the expression patterns of AIM2 inflammasome in cholesteatoma tissues. As the cytoplasmic dsDNA sensor $(15,16)$ and the first non-NLR family inflammasome, AIM2 has been reported to be present primarily in peripheral monocytes, and is also a significant node in the innate immune system (24). The AIM2 inflammasome can also be expressed and activated in certain tissue cells, such as keratinocytes $(18,25)$, neurons (26) and islet- $\beta$ cells (27). It was revealed in the present study that AIM2, ASC, caspase-1, IL-1 $\beta$ and IL-18 protein expression levels were notably increased in cholesteatoma tissues compared with healthy skin. These data suggested that the AIM2 inflammasome cascade may be activated in cholesteatoma tissues.

Collectively, the present study provided evidence that AIM2 is expressed in human cholesteatoma tissues and cultured cholesteatoma keratinocytes. Cholesteatomas are often categorized as congenital or acquired. Congenital cholesteatoma is referred to as the presence of an epithelial sac behind an intact tympanic membrane without a history of otitis media (28). Acquired cholesteatoma is often accompanied by chronic bacterial infection (1). The present study demonstrated that AIM2 was expressed at higher levels in cholesteatoma compared with that in external auditory canal skin. However, increased AIM2 expression levels were reported in acquired cholesteatoma tissues compared with congenital cholesteatoma tissues. As previous studies revealed that IFN- $\gamma$ is a strong inducer of AIM2 (25), the present study investigated the effect of IFN- $\gamma$ on the expression of AIM2 in cholesteatoma keratinocytes. Low basal titers of AIM2 protein were detected in cultured cholesteatoma keratinocytes, which were increased following IFN- $\gamma$ stimulation. Thus, a potential reason for the higher AIM2 expression levels in acquired cholesteatoma could be that the pro-inflammatory cytokines secreted by the inflammatory cells in the pre-matrix of acquired cholesteatoma tissues may influence the expression of AIM2.

In the present study, the protein expression levels of IL-1 $\beta$ and IL-18 were not statistically significantly different between 
A

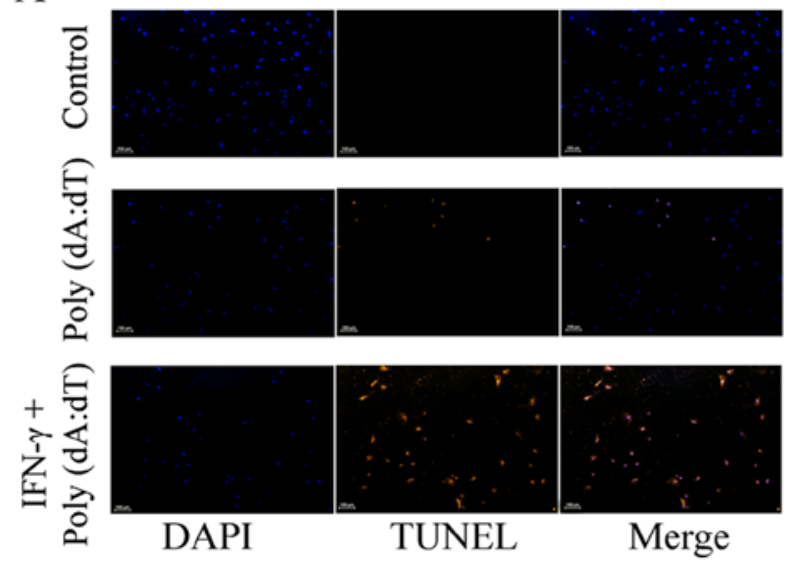

$\mathrm{C}$

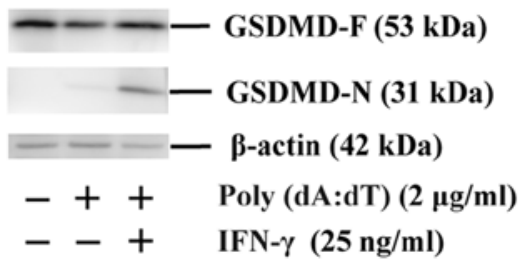

B

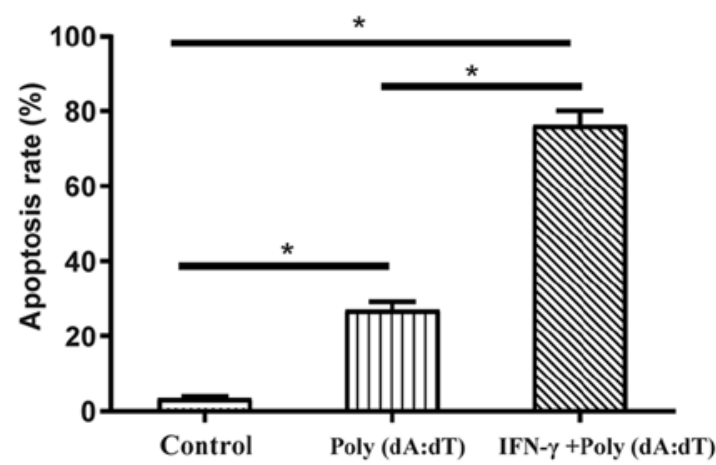

$\mathrm{D}$

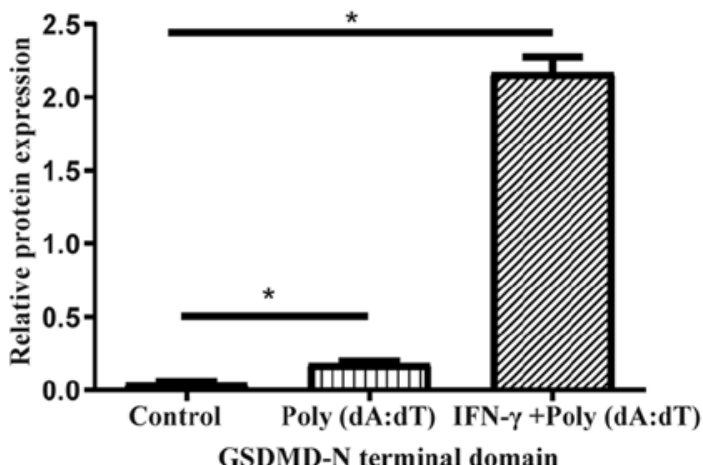

Figure 4. IFN- $\gamma$ stimulation promotes poly(dA:dT)-triggered cholesteatoma keratinocyte pyroptosis. (A and B) TUNEL assay demonstrated that poly(dA:dT) promoted apoptosis in the presence of IFN- $\gamma$. (C and D) Protein expression of GSDMD-F and GSDMD-N in cholesteatoma keratinocytes following stimulation with poly(dA:dT) alone, and IFN- $\gamma$ and poly $(\mathrm{dA}: \mathrm{dT})$ combined. Relative protein expression content was standardized to $\beta$-actin expression. Poly(dA:dT) enhanced the cleavage of GSDMD-N, but did not affect the expression of GSDMD-F. The presence of IFN- $\gamma$ resulted in greater protein expression levels of GSDMD-N. The columns indicate the mean \pm standard deviation values ( $\mathrm{n}=3$ per group). Data were acquired from three independent assays. ${ }^{*} \mathrm{P}<0.05$. GSDMD-F, full-length gasdermin D; GSDMD-N, terminal cleavage product of gasdermin D.

congenital and acquired cholesteatoma. One possible reason is that IL-1 $\beta$ and IL-18 were secreted into the extracellular matrix and were then degraded. Another possible reason is that the sample size was not large enough to be significant. Of course, there are other mechanisms that can induce the production of IL-1 $\beta$ and IL-18, such as bacterial or viral infection. However, the purpose of investigating cholesteatoma tissues was to confirm whether the target proteins, IL-1 $\beta$ and IL-18, which are regulated by AIM2, were expressed in cholesteatoma tissues.

Cytoplasmic DNA has been recognized as a danger signal that can be identified using AIM2 as a DNA sensor. For example, cytoplasmic dsDNA induces the initiation of the AIM2 inflammasome that leads to the production of IL-1 $\beta$ in human keratinocytes (25). In the present study, AIM2, ASC and caspase-1 expression levels increased following induction with poly(dA:dT), implying that AIM2 serves as a DNA sensor in the inflammatory response in cholesteatoma. A previous study reported that numerous extracellular DNA fragments were observed in the cholesteatoma epithelium (18). These findings suggested that cholesteatoma keratinocytes inherently express AIM2 and facilitate the activation of AIM2 inflammasomes in response to host-derived cytoplasmic DNA. Therefore, despite the upregulation of AIM2, caspase-1 and ASC, the expression levels of IL-18, cleaved-caspase-1 and IL-1 $\beta$ were increased following treatment with cytoplasmic DNA after priming with IFN- $\gamma$. These data demonstrated that dsDNA-triggered IL-1 $\beta$ and IL-18 secretion in cholesteatoma keratinocytes was mediated by the activated AIM2 inflammasome, which could be a pivotal mediator of inflammation in cholesteatoma.

The present study observed an increased percentage of TUNEL-positive cells, increased expression of GSDMD-N, and increased IL-1 $\beta$ and IL-18 production following stimulation of cholesteatoma keratinocytes with poly (dA:dT). These data indicated the loss of cell membrane integrity and increased cell pyroptosis of cholesteatoma keratinocytes. Furthermore, IFN- $\gamma$ was documented to markedly enhance poly(dA:dT)-triggered pyroptosis. Pyroptosis is a highly distinct type of inflammatory programmed cell death that is different from necrosis or apoptosis (22). Pyroptosis results in plasma membrane rupture and the secretion of damage-associated molecular pattern (DAMPs) biomolecules into the extracellular space (23). Thus, cell pyroptosis has been documented to lead to inflammation, chemotaxis of immune cells and further perpetuate the inflammatory cascade through release of various DAMPs, as well as pro-inflammatory mediators, consisting of IL-1 $\beta$, IL-18 and the alarmin high mobility group box $1(22,23)$. Also named IFN- $\gamma$-inducing factor, IL-18 induces IFN- $\gamma$ secretion (29). In 
addition, previous studies demonstrated that IL-1 may serve a vital role in the pathogenesis of acquired and congenital cholesteatomas by modulating bone resorption and cell proliferation $(1,10,17)$. Thus, the present study hypothesized that AIM2 inflammasome-mediated maturation and release of IL-1 $\beta$ and IL-18 from cholesteatoma keratinocytes both contribute to the immune response in cholesteatoma tissues.

In summary, the present study reported increased expression levels of inflammasome constituents (caspase-1, AIM2 and ASC) and their downstream cytokines (IL-18 and IL-1 $\beta$ ) in cholesteatoma tissues; these findings indicated the redundant activation of inflammasomes in cholesteatoma tissue. In addition, the results of the present study demonstrated that poly(dA:dT) induced AIM2 inflammasome activation in cholesteatoma keratinocytes, which increased cell pyroptosis and the production of IL-1 $\beta$ and IL-18. Pro-inflammatory cytokines (such as IFN- $\gamma$ ) could enhance the expression levels of AIM2 inflammasome constituents and promote poly(dA:dT)-induced cell death and bioactive cytokine production. These events demonstrated that the persistent expression of extracellular DNA fragments and the subsequent expression of AIM2 in cholesteatoma led to cell pyroptosis, as well as the production of inflammatory cytokines, which could cause bone resorption and perpetuate the inflammatory cascade in cholesteatoma.

\section{Acknowledgements}

The authors would like to thank Professor Shufeng Li (ENT Institute and Department of Otolaryngology, Eye and ENT Hospital, Fudan University, China) for helpful instruction and comments on the manuscript.

\section{Funding}

This work was funded by Science Project of Shanghai Municipal Health Bureau (grant no. 20174Y0062).

\section{Availability of data and materials}

The datasets used and/or analyzed during the current study are available from the corresponding author on reasonable request.

\section{Authors' contributions}

$\mathrm{CZ}$ and $\mathrm{MC}$ performed the experiments. $\mathrm{ZC}$ and $\mathrm{CZ}$ wrote and revised the manuscript. $\mathrm{CZ}$ and $\mathrm{MC}$ confirm the authenticity of all the raw data. $\mathrm{CZ}$ and $\mathrm{ZC}$ performed the statistical analysis. MC conducted the pathological diagnosis. All authors read and approved the final manuscript.

\section{Ethics approval and consent to participate}

Signed informed consent was obtained from all participants, if a patient was $<18$ years old, informed consent was obtained from a parent or guardian. Human studies conformed to the principles of the Declaration of Helsinki and were approved by the Research Ethics Committee of the Eye and ENT Hospital of Fudan University (approval no. 2017024).

\section{Patient consent for publication}

Not applicable.

\section{Competing interests}

The authors declare that they have no competing interests.

\section{References}

1. Olszewska E, Wagner M, Bernal-Sprekelsen M, Ebmeyer J, Dazert S, Hildmann $\mathrm{H}$ and Sudhoff H: Etiopathogenesis of cholesteatoma. Eur Arch Otorhinolaryngol 261: 6-24, 2004.

2. Si Y, Chen YB, Chen SJ, Zheng YQ, Liu X, Liu Y, Jiang HL, $\mathrm{Xu} \mathrm{G}, \mathrm{Li} \mathrm{ZH}$, Huang $\mathrm{QH}$, et al: TLR4 drives the pathogenesis of acquired cholesteatoma by promoting local inflammation and bone destruction. Sci Rep 5: 16683, 2015.

3. Inagaki T and Paparella MM: Chronic otitis media with cholesteatoma: Middle ear/inner ear interaction. Otol Neurotol 30: 430-431, 2009.

4. Grundfast KM, Ahuja GS, Parisier SC and Culver SM: Delayed diagnosis and fate of congenital cholesteatoma (keratoma). Arch Otolaryngol Head Neck Surg 121: 903-907, 1995.

5. Tlaskalová-Hogenová $H$, Tucková L, Lodinová-Zádniková $\mathrm{R}$, Stepánková R, Cukrowska B, Funda DP, Striz I, Kozáková H, Trebichavský I, Sokol D, et al: Mucosal immunity: Its role in defense and allergy. Int Arch Allergy Immunol 128: 77-89, 2002.

6. Chang JH, McCluskey PJ and Wakefield D: Toll-like receptors in ocular immunity and the immunopathogenesis of inflammatory eye disease. Br J Ophthalmol 90: 103-108, 2006.

7. Kumar A, Hazlett LD and Yu FS: Flagellin suppresses the inflammatory response and enhances bacterial clearance in a murine model of Pseudomonas aeruginosa keratitis. Infect Immun 76: 89-96, 2008.

8. Kawai T and Akira S: TLR signaling. Cell Death Differ 13: 816-825, 2006

9. Lee MS and Kim YJ: Pattern-recognition receptor signaling initiated from extracellular, membrane, and cytoplasmic space. Mol Cells 23: 1-10, 2007.

10. Jiang H, Si Y, Li Z, Huang X, Chen S, Zheng Y, Xu G, Chen X, Chen Y, Liu Y, et al: TREM-2 promotes acquired cholesteatoma-induced bone destruction by modulating TLR4 signaling pathway and osteoclasts activation. Sci Rep 6: 38761, 2016.

11. Leichtle A, Klenke C, Ebmeyer J, Daerr M, Bruchhage KL, Hoffmann AS, Ryan AF, Wollenberg B and Sudhoff H: NOD-Like Receptor Signaling in Cholesteatoma. BioMed Res Int 2015: 408169, 2015.

12. Kariya S, Okano M, Zhao P, Kataoka Y, Yoshinobu J, Maeda Y, Ishihara H, Higaki T and Nishizaki K: Activation of NLRP3 inflammasome in human middle ear cholesteatoma and chronic otitis media. Acta Otolaryngol 136: 136-140, 2016.

13. Strowig T, Henao-Mejia J, Elinav E and Flavell R: Inflammasomes in health and disease. Nature 481: 278-286, 2012.

14. Lamkanfi $M$ and Dixit VM: Inflammasomes and their roles in health and disease. Annu Rev Cell Dev Biol 28: 137-161, 2012.

15. Fernandes-Alnemri T, Yu JW, Datta P, Wu J and Alnemri ES: AIM2 activates the inflammasome and cell death in response to cytoplasmic DNA. Nature 458: 509-513, 2009.

16. Hornung V, Ablasser A, Charrel-Dennis M, Bauernfeind F, Horvath G, Caffrey DR, Latz E and Fitzgerald KA: AIM2 recognizes cytosolic dsDNA and forms a caspase-1-activating inflammasome with ASC. Nature 458: 514-518, 2009.

17. Chi Z, Wang Z, Liang Q, Zhu Y and du Q: Induction of cytokine production in cholesteatoma keratinocytes by extracellular high-mobility group box chromosomal protein 1 combined with DNA released by apoptotic cholesteatoma keratinocytes. Mol Cell Biochem 400: 189-200, 2015.

18. Kopfnagel V, Wittmann M and Werfel T: Human keratinocytes express AIM2 and respond to dsDNA with IL-1 $\beta$ secretion. Exp Dermatol 20: 1027-1029, 2011.

19. de Koning HD, Bergboer JG, van den Bogaard EH, van Vlijmen-Willems IM, Rodijk-Olthuis D, Simon A, Zeeuwen PL and Schalkwijk J: Strong induction of AIM2 expression in human epidermis in acute and chronic inflammatory skin conditions. Exp Dermatol 21: 961-964, 2012. 
20. Derlacki EL and Clemis JD: Congenital cholesteatoma of the middle ear and mastoid. Ann Otol Rhinol Laryngol 74: 706-727, 1965.

21. Huang S, Song Z, Jiang L, Chen L, Wang R, Qin W, Liu P and Lin Z: Absent in melanoma 2 (AIM2) expressed in human dental pulp mediates IL-1 $\beta$ secretion in response to cytoplasmic DNA. Inflammation 38: 566-575, 2015.

22. Xu YJ, Zheng L, Hu YW and Wang Q: Pyroptosis and its relationship to atherosclerosis. Clin Chim Acta 476: 28-37, 2018.

23. Zhang Y, Chen X, Gueydan C and Han J: Plasma membrane changes during programmed cell deaths. Cell Res 28: 9-21, 2018.

24. Guo Q, Wu Y, Hou Y, Liu Y, Liu T, Zhang H, Fan C, Guan H, Li Y, Shan Z, et al: Cytokine secretion and pyroptosis of thyroid follicular cells mediated by enhanced NLRP3, NLRP1, NLRC4, and AIM2 inflammasomes are associated with autoimmune thyroiditis. Front Immuno 9: 1197, 2018.

25. Dombrowski Y, Peric M, Koglin S, Kammerbauer C, Göss C, Anz D, Simanski M, Gläser R, Harder J, Hornung V, et al: Cytosolic DNA triggers inflammasome activation in keratinocytes in psoriatic lesions. Sci Transl Med 3: 82ra38, 2011.
26. Kaushal V, Dye R, Pakavathkumar P, Foveau B, Flores J, Hyman B, Ghetti B, Koller BH and LeBlanc AC: Neuronal NLRP1 inflammasome activation of Caspase-1 coordinately regulates inflammatory interleukin-1-beta production and axonal degeneration-associated Caspase- 6 activation. Cell Death Differ 22: 1676-1686, 2015.

27. Oslowski CM, Hara T, O'Sullivan-Murphy B, Kanekura K, Lu S, Hara M, Ishigaki S, Zhu LJ, Hayashi E, Hui ST, et al: Thioredoxin-interacting protein mediates ER stress-induced $\beta$ cell death through initiation of the inflammasome. Cell Metab 16: 265-273, 2012.

28. Bhutta MF, Williamson IG and Sudhoff HH: Cholesteatoma. BMJ 342: d1088, 2011.

29. Novick D, Kim S, Kaplanski G and Dinarello CA: Interleukin-18, more than a Th1 cytokine. Semin Immunol 25: 439-448, 2013.

(i) $\Theta$ This work is licensed under a Creative Commons Attribution-NonCommercial-NoDerivatives 4.0 International (CC BY-NC-ND 4.0) License. 